\title{
Effect of Spacecraft Aerodynamics and Heat Shield Characteristics on Optimal Aeroassisted Transfer
}

\author{
Antonio Mazzaracchio, Mario Marchetti \\ Astronautical, Electrical, and Energetic Engineering Department, Sapienza University of Rome, Rome, Italy \\ Email: a_mazzaracchio@hotmail.com
}

Received March 6, 2012; revised April 16, 2012; accepted April 24, 2012

\begin{abstract}
A spacecraft designed to operate in a planetary atmosphere must have an adequate heat shield to withstand the high heat fluxes and heat loads that are generated by aerodynamic heating. Very often, the mass of the thermal protection system is a significant fraction of the total mass of the vehicle. In contrast, performing maneuvers in the atmosphere, that would be very costly in terms of propellant consumption if they were performed completely outside of the atmosphere in a classic way, is a very attractive prospective technique. The advantages and disadvantages in terms of total mass spared must be determined. The mission investigated involves an aeroassisted coplanar transfer from a high to a low Earth orbit. The approach uses a combination of three propulsive impulses in space together with an aerodynamic maneuver in the atmosphere. The heat shield adopted is fully ablative, given the expected high values of the entering heat flux. The convenience of the aeroassisted maneuver and the influence of the parameters involved are evaluated in comparison to a conventional Hohmann transfer. In particular, a parametric analysis is performed by varying the following characteristics of the vehicle: aerodynamic efficiency, mass-to-surface ratio, deorbit impulse, and initial altitude of the orbit. The influence of the thermal protection system is examined by assessing the impact of the type of ablative material employed, the thermal safety factor, and the allowable temperature for the adhesive layer on the substructure. The analysis is conducted with a highly representative thermal model by coupling the dynamic and thermal analyses and using a genetic optimizer. The optimization methodology and the thermal model are completely original. The results indicate the importance of choosing low-density ablative materials, of adopting a suitable thermal safety factor, and of choosing high-performance adhesives. The optimal trajectories obtained correspond to a zero second propulsive impulse.
\end{abstract}

Keywords: Aeroassisted Maneuver; Heat Shield; Optimization; Orbital Transfer; Thermal Protection System

\section{Introduction}

One of the future objectives in space activities is to use aeroassisted orbital maneuvers, i.e., maneuvers that are carried out with the aid of the atmosphere, to satisfy the increasingly stringent constraints of the cost of space missions. Depending on the goal of the mission, aeroassisted maneuvers are performed in different ways: aerogravity assist, aerobraking, and aerocapture. To these maneuvers, one must add the re-entry maneuvers and some possible variants such as the skip re-entry technique. These maneuvers are very attractive, although they are only theoretical at this point (except for rare uses in aerobraking), because they can substantially reduce the requirements of a space mission in terms of propulsion and flight time in favor of, among other things, the possibility of housing a larger payload.

Underlying this approach is the possibility of exploiting the presence of the atmosphere of the celestial body around which one wants to operate to lower the overall energy required. In practice, one tries to execute the com- plete operation with the help of aerodynamics because orbital maneuvers are quite expensive in terms of propulsion, especially those outside the orbital plan. Thus, the design of a spacecraft with specific reference to atmospheric portion of flight must present an efficient aerodynamic configuration; however, the priority constraint of the overall cost of space missions must be satisfied.

From this brief introduction, it is already clear that once shown the technical feasibility of the aeroassisted maneuver, one must evaluate its convenience compared with alternative hypotheses, such as a classical purely propulsive maneuver. In fact, optimizing a spacecraft and its mission, or more specifically its trajectory for a given mission, is always a compromise between the interests of performance, security, and economics, which are almost always in mutual conflict.

Usually, minimizing the mass of the heat shield while respecting the limits of safety is a primary requirement. Indeed, any savings in terms of the mass of the thermal protection system (TPS) can be translated into an increase 
of the "useful" mass, namely, into an opportunity to accommodate a greater payload or extend other spacecraft subsystems. The problem becomes more complex when considering an aeroassisted maneuver and taking into account the mass of propellant still required during some of the various phases of the maneuver itself.

In this regard, a basic scheme is usually adopted in the literature to perform an aeroassisted maneuver-the use of propulsion only in space together with one or more atmospheric segments of pure aerodynamic flight - which is also considered in this work. Maximizing the benefits at the propulsive level for an aeroassisted maneuver normally requires a more intensive use of the atmospheric phase of flight. Thus, the resulting trajectory touches the denser layers of the atmosphere with longer crossing times. Therefore, a larger TPS with its relative higher mass fraction must be adopted.

A legitimate question that arises at this point is whether the resulting increase in the mass of the TPS, together with the mass of propellant required to enter the atmosphere and then to achieve the final orbit, may override the convenience of the aeroassisted maneuver compared with a classical operation, which is a purely propulsive extra-atmospheric maneuver. It seems evident that this is an optimization problem.

All of the above considerations concerning both the evaluation of the convenience of the aeroassisted approach and the search for the optimal solution and the methodological sphere itself are the basis of the research questions that motivated this work.

An original procedure was carried out jointly with the implementation of software developed by the authors to optimize the aeroassisted orbital maneuvers using a genetic algorithm (GA) with simultaneous evaluation of the optimal configuration of the associated heat shields and the coupling of the dynamic and thermal analyses. The tool was verified by comparing its results with those found in the literature. This tool, because of its level of implementation details, is suitable for the conceptual development stages of a spacecraft and its mission.

The initial intention was to evaluate the influence of various parameters - orbital, aerodynamic, and dimensional - on the feasibility and convenience of the mission. For an introductory analysis of the problem and to evaluate the importance of various factors, coplanar transfer (aerobraking) from a high Earth orbit (HEO) to a low Earth orbit (LEO), both circular, is proposed as a case study. The vehicle is a delta wing spacecraft that is protected by an ablative TPS with uniform thickness. The problem is studied in the absence of constraints on the maximum allowable entering heat flux.

Beyond the presentation of the problem in Section 1, Section 2 describes the model and the optimization procedure. The case study is presented in Section 3, and the relevant results and analyses are discussed in Section 4. Finally, Section 5 offers a summary, conclusions, and recommendations for future improvements.

\section{Models and Optimization}

The description of the models, the governing equations, and the relevant assumptions for the problem-i.e., thermal models, aerodynamic heating, atmospheric flight mechanics, and heat shield configuration - are thoroughly presented in [1] and [2]. Reference [2] can be consulted for full details of the original optimization procedure.

All of the analysis presented here was performed using the cited software developed by the authors called ATHSHO (Aeroassisted Trajectory and Heat SHield Optimization). It is important to recall that thermal analysis is performed with a one-dimensional plane model and that the adopted GA refers to a mixed one-point/two-point crossover operator together with a reproduction plan that provides a full generational replacement with elitism [3-5].

\section{Case Study}

A parametric analysis was performed by varying the following characteristics of the vehicle: aerodynamic efficiency, mass-to-surface ratio, deorbit impulse, and the altitude of the initial orbit. The influence of the characteristics of the TPS was examined by assessing the type of ablative material adopted, the thermal safety factor, and the allowable temperature for the bond-line, i.e., the adhesive layer on the substructure.

\subsection{Hypotheses}

The main hypotheses considered are the following:

- The initial total mass of the vehicle is given.

- The attitude control is accomplished only through the angle of attack.

- The entering heat flux is unconstrained.

- The TPS is fully ablative with uniform thickness.

\subsection{Vehicle}

The vehicle model is a delta wing shuttle with a high $L / D$ ratio, which is comparable in the first instance to the configuration and dimensions of the Boeing X-37A vehicle (Figure 1).

The dimensions, sizes, and aerodynamic characteristics of the vehicle used were taken in part from [6-8]. Other data were reasonable assumptions made by the authors. The main dimensions and characteristics of the vehicle are listed in Table 1, and the principal aerodynamic and propulsive parameters are listed in Table 2.

The values shown are those considered for the nominal reference case. Some of them may vary depending on the purpose of the study (Section 4). 


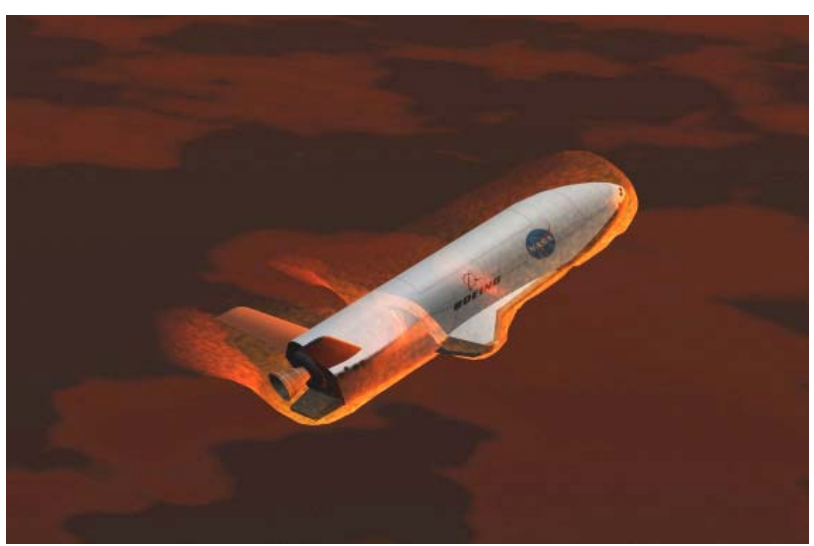

Figure 1. Boeing X-37A (source NASA.gov).

Table 1. Vehicle dimensions and characteristics.

\begin{tabular}{ccc}
\hline Vehicle length & $l_{v e}$ & $9.38 \mathrm{~m}$ \\
Vehicle body radius & $r_{b}$ & $1.00 \mathrm{~m}$ \\
Vehicle wing span & $w s_{v e}$ & $4.50 \mathrm{~m}$ \\
Vehicle wing cord & $w c_{v e}$ & $3.50 \mathrm{~m}$ \\
Vehicle reference surface & $S$ & $11.69 \mathrm{~m}^{2}$ \\
Vehicle TPS total surface & $S_{T P S, v e}$ & $42.65 \mathrm{~m}^{2}$ \\
Bond-line limit temperature & $T_{B L, \text { lim }}$ & $450 \mathrm{~K}$ \\
Thermal safety factor & $T S F$ & 1 \\
\hline
\end{tabular}

Table 2. Vehicle's aerodynamic and propulsive characteristics.

\begin{tabular}{ccc}
\hline Zero-lift drag coefficient & $C_{D 0}$ & 0.032 \\
Induced drag factor & $K_{D}$ & 1.4 \\
Lift coefficient derivative & $C_{L, \alpha}$ & 0.5699 \\
Maximum lift coefficient & $C_{L, \max }$ & 0.4 \\
Propellant specific impulse & $I_{s p}$ & $310 \mathrm{~s}$ \\
\hline
\end{tabular}

\subsection{Mission and Maneuver}

Table 3 lists the values of the altitudes for the initial Geostationary Earth Orbit (GEO) and final LEO for the aerobraking maneuver as well as the conventional height assumed for the atmosphere.

Even in this case, the indicated values are those used for the reference case. In particular, in the parametric analysis performed, the value of the initial HEO altitude was varied. The physical properties of the atmosphere were derived from the model 1976 US Standard Atmosphere [9].

Figure 2 shows a classic schematic for a HEO-LEO aerobraking maneuver. The strategy involves the combined use of aerodynamic maneuvering in the atmosphere and some extra-atmospheric propulsion phases. More precisely, one assumes that the propulsive phases are concentrated in three impulses in space and that the portion of atmospheric flight is performed without the
Table 3. Maneuver characteristics.

\begin{tabular}{llc}
\hline Initial HEO (GEO) altitude & $H_{A}$ & $35,786 \mathrm{~km}$ \\
Final LEO altitude & $H_{B}$ & $480 \mathrm{~km}$ \\
Atmosphere's upper limit & $H_{a t m}$ & $129.6 \mathrm{~km}$ \\
\hline
\end{tabular}

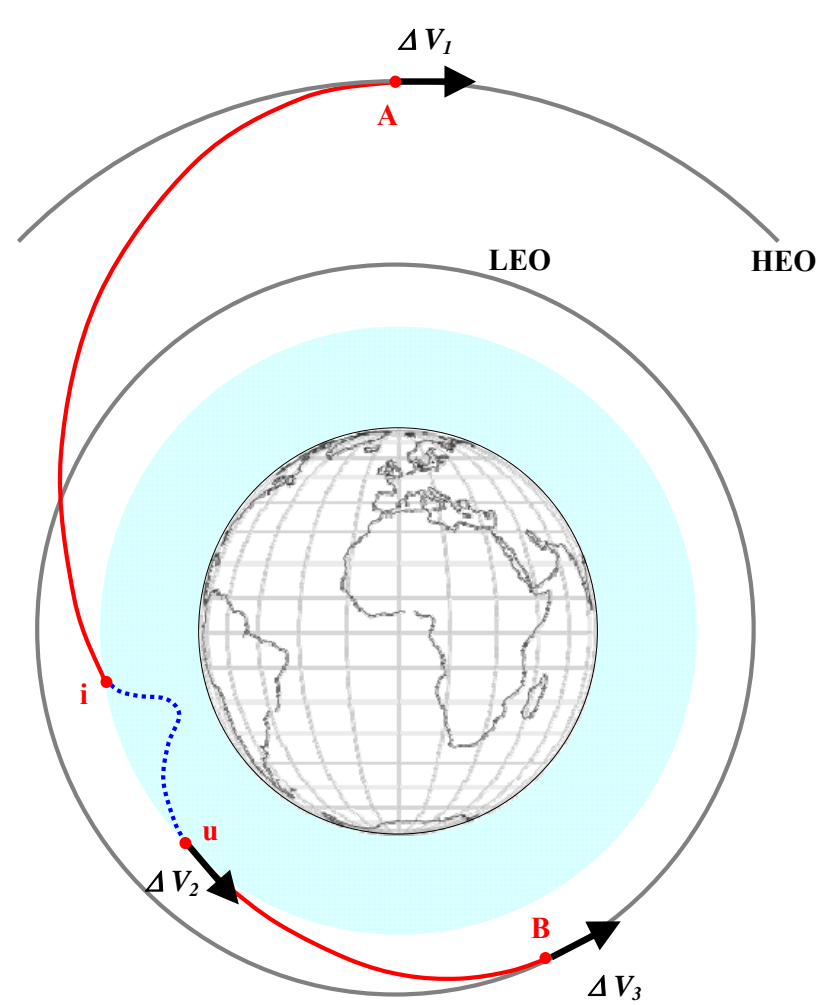

Figure 2. Schematic of a HEO-LEO aerobraking maneuver.

use of the propulsion system. The first propulsive impulse (deorbit) at the altitude $H_{A}$ of the initial HEO varies the vehicle's speed by $\Delta V_{1}$ to enter the atmosphere along an elliptic orbit segment. The second impulse (boost) is applied upon the exit from the atmosphere to achieve the final LEO by ascending once more along an elliptic orbit segment. The boost is expressed by a speed variation of the vehicle, $\Delta V_{2}$.

The third and final impulse (circularizing) varies the speed by $\Delta V_{3}$ to circularize the vehicle's path within the altitude $H_{B}$ of the final LEO.

It is useful at this point to describe the various phases of the maneuver in more detail as follows. Initially, the vehicle is moving on a circular orbit of radius $R_{A}$ with a speed $V_{A}$ around the Earth, which has radius $R_{\oplus}$. The expression of the circular speed is the following:

$$
V_{A}=\sqrt{\mu / R_{A}}
$$

where

$$
R_{A}=R_{\oplus}+H_{A} .
$$

The deorbit is accomplished by applying the first impulse $\Delta V_{1}$ in the opposite direction of the spacecraft's 
speed. This impulse puts the vehicle along an elliptic orbit with the perigee inside the dense layers of the atmosphere.

The atmospheric region below the altitude $H_{a t m}$ in which the aerodynamic effects are considered to be conventionally present is denoted the sensible atmosphere. One can determine the speed $V_{i}$ and the flight path angle $\gamma_{i}$ of the vehicle's trajectory at the atmospheric entry point ( $\left.H=H_{a t m}\right)$ through the following relationships, which are obtained according to energetic considerations:

$$
\begin{gathered}
V_{i}=\sqrt{2 \mu\left(\frac{1}{R_{a t m}}+\frac{\left(V_{A}-\Delta V_{1}\right)^{2}}{2}-\frac{1}{R_{A}}\right)} \\
\gamma_{i}=\cos ^{-1}\left[\frac{R_{A}}{R_{a t m} V_{i}}\left(V_{A}-\Delta V_{1}\right)\right]
\end{gathered}
$$

where

$$
R_{\text {atm }}=R_{\oplus}+H_{\text {atm }} .
$$

Clearly, it is necessary that the applied $\Delta V_{1}$ be greater than the minimum $\Delta V_{1, \min }$ for which there would be only a tangential trajectory to the edge of the sensible atmosphere. This $\Delta V_{1, \min }$ is presented in Figure 3 and given by the following expression:

$$
\Delta V_{1, \text { min }}=\sqrt{\frac{\mu}{R_{A}}}-\sqrt{2 \mu \frac{\frac{1}{R_{\text {atm }}}-\frac{1}{R_{A}}}{\left(\frac{R_{A}}{R_{\text {atm }}}\right)^{2}-1} .}
$$

During the atmospheric portion of flight, the vehicle performs the required maneuver, which is optimally controlled by modulations of the angle of attack $\alpha$ (leaving the bank angle $\sigma$ fixed) subject to the heating constraints, if any. During this phase, the vehicle's speed decreases because of aerodynamic drag, and because of this loss of energy, a new impulse is necessary to achieve the final altitude.

At the end of atmospheric flight, the vehicle is situated at an altitude $H_{a t m}$ again, is driven at a speed $V_{u}$, and has a flight path angle equal to $\gamma_{u}$. At this moment, a boost impulse is applied to enter an ascending elliptic orbit with the apogee equal to the radius of the final circular orbit.

The required $\Delta V_{2}$, as a function of $V_{u}$ and $\gamma_{u}$, can be found from the following expression:

$$
\Delta V_{2}=\sqrt{\frac{\frac{1}{R_{B}}-\frac{1}{R_{a t m}}}{1-\left(\frac{R_{B}}{R_{a t m}}\right)^{2} \cos ^{2} \gamma_{u}}}-V_{u}
$$

where

$$
R_{B}=R_{\oplus}+H_{B} .
$$

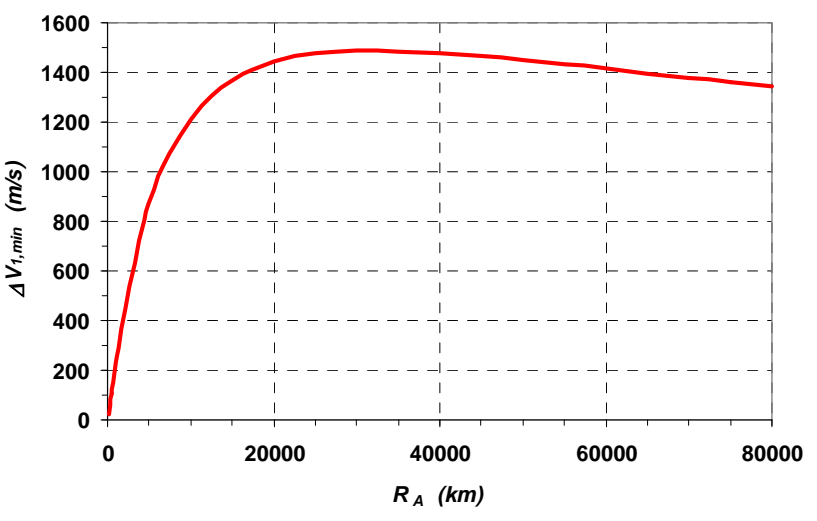

Figure 3. $\Delta V_{\mathrm{l}, \min }$ vs initial HEO radius.

Once the final altitude is reached, the third impulse is applied to circularize the final orbit. The expression for the third $\Delta V$ is the following:

$$
\Delta V_{3}=\sqrt{\frac{\mu}{R_{B}}}-\sqrt{2 \mu \frac{\frac{1}{R_{B}}-\frac{1}{R_{a t m}}}{1-\left(\frac{R_{B}}{R_{a t m}}\right)^{2} \cos ^{2} \gamma_{u}}} \cdot \frac{R_{B}}{R_{a t m}} \cos \gamma_{u} .
$$

\subsection{Fitness Function, Objective Function, and Constraints}

The total initial mass of the vehicle is the sum of the propellant mass, the TPS mass, and the structural and payload masses. The goal of the current optimization problem is to perform the assigned orbital transfer while minimizing the sum of the mass of the propellant and the mass of the TPS needed. The objective function, which must be maximized, is then given by the final mass of the vehicle, which can be defined as the performance index of the problem. There are some events that cause variation in the mass of the vehicle. The first is the consumption of fuel due to the deorbit impulse; thus, one can calculate the mass of the vehicle entering the atmosphere. The latter can be derived directly using the Tsiolkovsky equation for the impulse in question:

$$
m_{v e, i}=m_{v e, i n i} e^{-\frac{\left|\Delta V_{1}\right|}{g_{0} I_{s p}}} .
$$

This mass is further reduced by $m_{T P S, l o s}$ during the passage through the atmosphere because of TPS ablation. The mass loss due to ablation comes from both the surface recession and the material density change due to pyrolysis. Thus, the mass of the vehicle at the atmosphere exit is the following:

$$
m_{v e, u}=m_{v e, i}-m_{T P S, l o s} .
$$

At this point, the boost and circularization impulses are applied in sequence, and the final vehicle mass is obtained by two successive applications of the Tsiolkovsky equation: 


$$
m_{v e, f i n}=m_{v e, u} e^{-\frac{\left|\Delta V_{2}\right|+\left|\Delta V_{3}\right|}{g_{0} I_{s p}}} .
$$

Compliance with the eventual constraint on the entering heat flux is ensured through a reward factor that is added to the objective function ( $m_{v e, \text { fin }} / m_{v e, \text { ini }}$ ) by means of an appropriate multiplicative weight $w_{H F}$, which is chosen specifically to achieve rapid convergence. Likewise, the objective function is multiplied by its weight $w_{m}$. Thus, the final expression for the fitness function for the genetic optimizer is as follows:

$$
f f=w_{m} \frac{m_{v e, f i n}}{m_{v e, i n i}}+w_{H F} R_{f, H F} .
$$

In this case study, the heat flux is considered unconstrained, and assuming $w_{m}=1$, the previous expression reduces to the following:

$$
f f=\frac{m_{v e, f i n}}{m_{v e, i n i}} .
$$

That is, the fitness function coincides with the objective function.

\subsection{Assessment of the Convenience}

The convenience of the aeroassisted maneuver can be assessed with respect to an equivalent Hohmann transfer. Such a transfer is completely "all propulsive" outside the atmosphere and based on two impulsive velocity changes at two points with radii $R_{A}$ and $R_{B}$, respectively:

$$
\begin{gathered}
\Delta V_{A}=\sqrt{\frac{\mu}{R_{A}}}\left(\sqrt{\frac{2 R_{B}}{R_{A}+R_{B}}}-1\right) \\
\Delta V_{B}=\sqrt{\frac{\mu}{R_{B}}}\left(1-\sqrt{\frac{2 R_{A}}{R_{A}+R_{B}}}\right) .
\end{gathered}
$$

Thus, the total "all propulsive" impulse is:

$$
\Delta V_{a p}=\left|\Delta V_{A}\right|+\left|\Delta V_{B}\right| \text {. }
$$

Consequently, the expression for the final mass of the vehicle in the case of the Hohmann transfer is as follows:

$$
m_{v e, f i n, a p}=m_{v e, i n i} e^{-\frac{\left|\Delta V_{a p}\right|}{g_{0} I_{s p}}} .
$$

\section{Results and Analysis}

The case study for the HEO-LEO coplanar transfer through aerobraking, which was described in the previous Section 3 , has been addressed by parameterising the mass-tosurface ratio $M / S$ of the vehicle, the $\Delta V_{1}$ corresponding to the deorbit impulse applied, the aerodynamic efficiency $\varepsilon$ (with $C_{L \text {, max }}$ fixed), the ablative material used, the maximum allowable temperature for the bond- line $T_{B L, \lim }$, and the thermal safety factor $T S F$. In addition, the above parametric analysis was performed for different values of the initial HEO altitude, which are expressed as fractions of the GEO.

More specifically, the following values were adopted:

- HEO Altitude: $35,786 \mathrm{~km}$ (=GEO); 53,679 km (HEOH $=1.5 \mathrm{GEO}) ; 17,893 \mathrm{~km}(\mathrm{HEOL}=0.5 \mathrm{GEO})$. The altitude for the final LEO is the same for all cases and equal to $480 \mathrm{~km}$.

- $M / S: 200 \mathrm{~kg} / \mathrm{m}^{2}, 300 \mathrm{~kg} / \mathrm{m}^{2}$ and $450 \mathrm{~kg} / \mathrm{m}^{2}$, corresponding, respectively, to low, medium and high load values. Incidentally, $300 \mathrm{~kg} / \mathrm{m}^{2}$ is the value found most frequently in the literature. These load values correspond to the initial mass of the vehicle: 2338.0 $\mathrm{kg}, 3507.0 \mathrm{~kg}$, and $5260.5 \mathrm{~kg}$.

- $\Delta V_{1}$ range: the range of the parametric first variation of $V$ (in steps of $10 \mathrm{~m} / \mathrm{s}$ ) between the minimum value (rounded to the nearest ten higher) given by Equation (6) and the maximum value that allows a solution to complete the aeroassisted maneuver. The minimum values of the deorbit impulse for the three HEO altitudes analyzed are (Figure 3) $1490 \mathrm{~m} / \mathrm{s}$ for GEO, $1440 \mathrm{~m} / \mathrm{s}$ for $\mathrm{HEOH}$, and $1420 \mathrm{~m} / \mathrm{s}$ for HEOL.

- $\varepsilon: 1.5$ and 3 correspond, respectively, to a medium and a high aerodynamic efficiency. These values were obtained by varying $C_{D 0}$ and $K_{D}$.

- $T F S: 1$ and 2 correspond, respectively, to a minimum nominal thickness of the TPS and a "safer" double thickness.

- $T_{B L, \lim }: 176.85^{\circ} \mathrm{C}(450 \mathrm{~K})$ and $400^{\circ} \mathrm{C}(673.15 \mathrm{~K})$ were both acquired from data in the literature; the first is used for standard adhesives, and the second is used for high-performance adhesives.

- Existing adhesives with enhanced characteristics allow the threshold temperature to be increased to $400^{\circ} \mathrm{C}$, which results in greater heat shield thickness savings [10].

- Ablative material: PICA-15 (hereinafter referred to as PICA), AVCOAT 5026-HCG (hereinafter referred to as AVCOAT), and FM 5055 CP in its Reduced Density version (hereinafter referred to as RDCP).

Among all the possible combinations of these parameters with their associated values, 12 cases were analyzed for each value of the $M / S$ ratio for a total of 36 different scenarios. The combinations analyzed are presented in Tables 4-6, where the index $(a, b$, and $c)$ marks the adopted value for the load. Moreover, because the range of variation of $\Delta V_{1}$ is analyzed by steps of $10 \mathrm{~m} / \mathrm{s}, 390$ different cases form the database of results for the analysis of the case study in question.

As a reference, the nominal case is chosen for the GEOHEO transfer that provides a TPS made of PICA, with $T_{B L, \lim }=450 \mathrm{~K}, T S F=1$, and $\varepsilon=1$. The twelve combinations chosen and the corresponding purpose of 
Table 4. HEO-LEO transfer, parameters and values (cases 1, 2, 3, and 4).

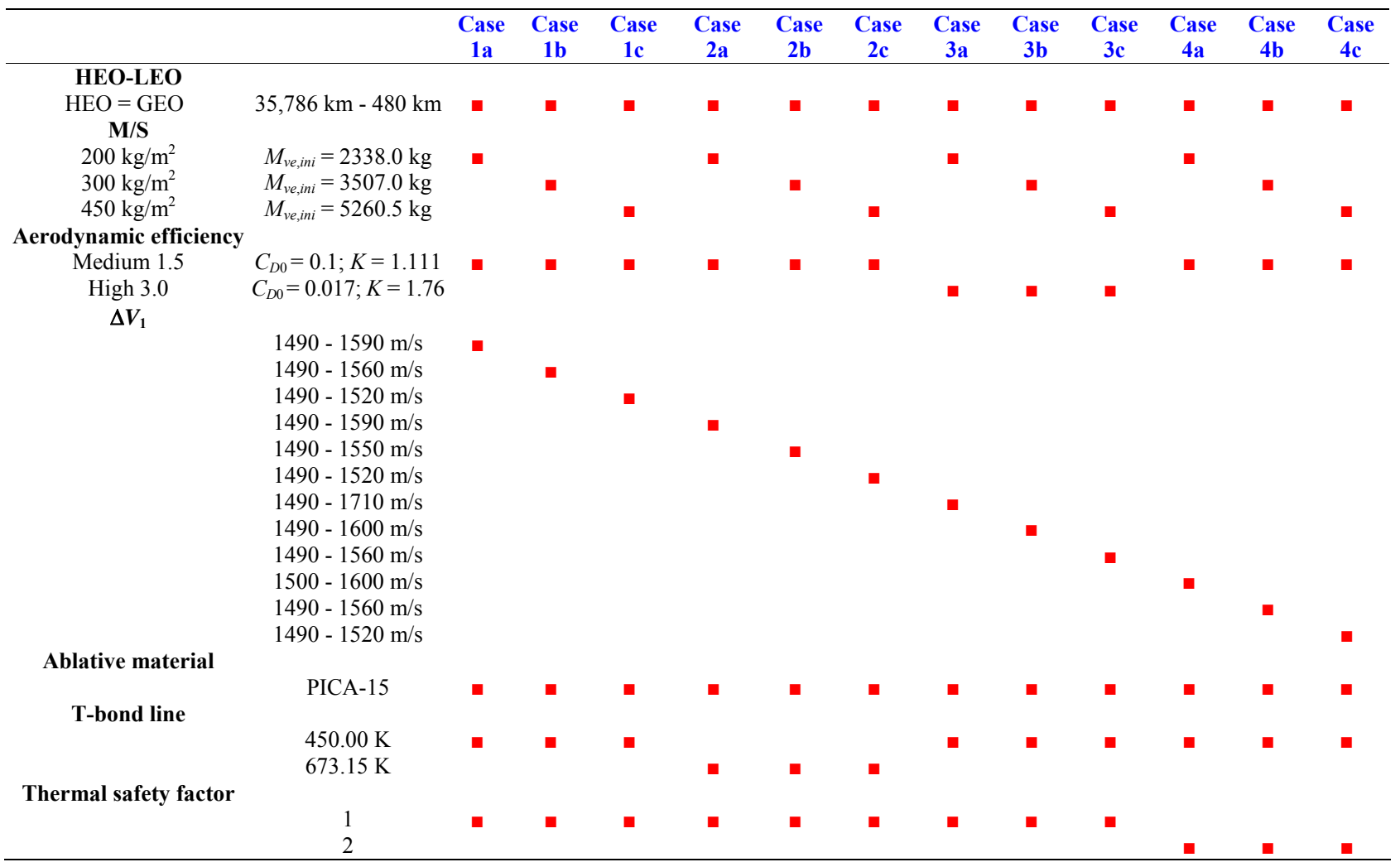

Table 5. HEO-LEO transfer, parameters and values (cases 5, 6, 7, and 8).

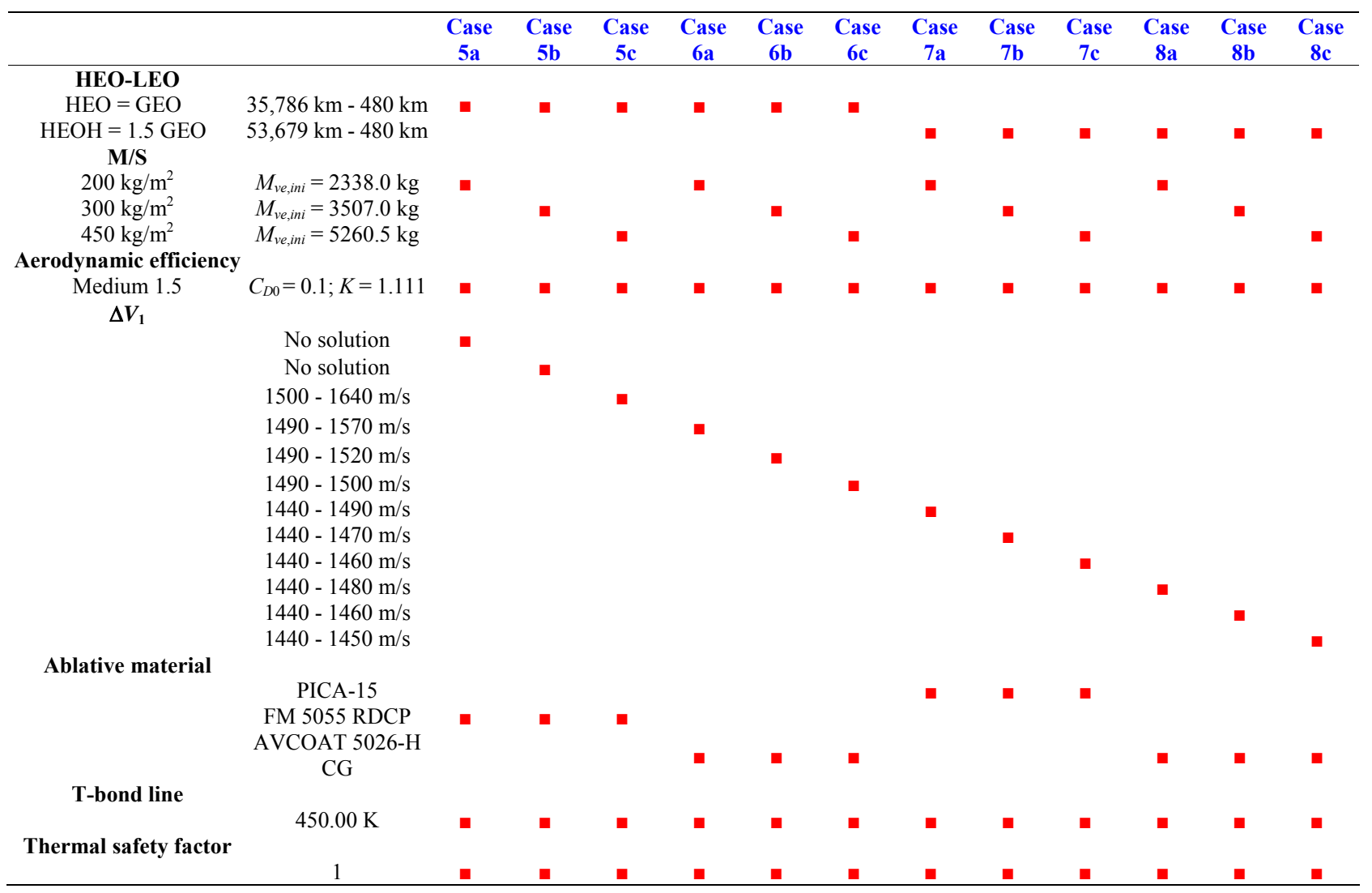


Table 6. HEO-LEO transfer, parameters and values (cases 9, 10, 11, and 12).

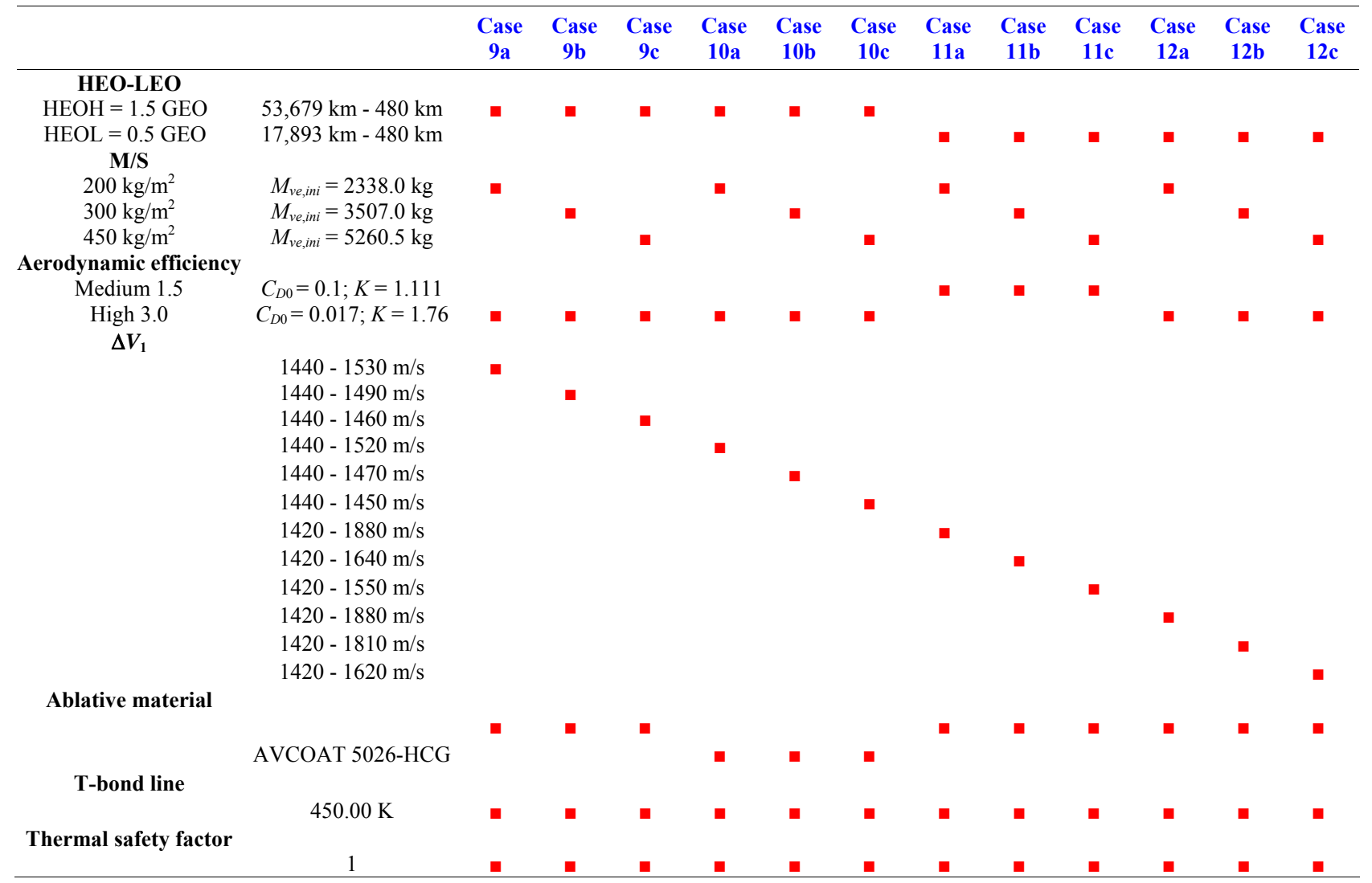

the evaluation are summarized in Table 7. Figures 4 to 15 report the trends of the mass gain compared with the "all propulsive" case with varied applied $\Delta V_{1}$ and parameterized as a function of the three chosen values of $M / S$, which were analyzed for each scenario.

The graph on the left of each figure shows the cited mass gain in absolute terms, whereas the graph on the right presents its performance in terms of the percentage of the initial vehicle's mass.

The mass gain is therefore a measure of the convenience of the aeroassisted maneuver compared with the corresponding Hohmann transfer. When reading these graphics, the definitions of the "range of feasibility" and the "range of convenience" are introduced.

The first one is the interval of $\Delta V_{1}$ in which the aeroassisted maneuver is feasible, whereas the second $\Delta V_{1}$ interval is the range in which the aeroassisted operation is more convenient than the "all propulsive" one. As mentioned above, having made the run for all values of $\Delta V_{1}$ leading to a solution, the range of feasibility is directly readable as the $\mathrm{x}$-axis interval of the definition of the curves themselves.

The range of convenience is by definition the interval in which the curve takes positive values. The range of convenience is always within the range of feasibility. Table 8 shows, for the value $M / S=200 \mathrm{~kg} / \mathrm{m}^{2}$ (case "a" of Tables 4-6), the values of the three propulsive impulses applied in each scenario, which in each case give the highest convenience for the maneuver (maxima of the "blue" curves in the graphs of Figures 4 to 15).

It is evident that the utmost convenience for the aeroassisted maneuver corresponds to a value of $\Delta V_{2}$ that is very close to zero, i.e., in the absence of the boost impulse (case 5a has no feasible solutions for the considered value for the load).

This outcome is valid in general for the other values of $M / S$. Thus, the trajectory optimization process tends to reduce the vehicle's speed while exiting the atmosphere to the "right" value to reach the final LEO with minimal energy expenditure. Saving this fuel for the second impulse is the basis for the achievement of the highest convenience.

Comparing Figures 4 to 15, one can draw some interesting conclusions:

- The increase of $M / S$ in all cases involves a decrease in the range of feasibility; in particular, the maximum possible $\Delta V_{1}$ diminishes (the right bound of the range).

- The increase of $T_{B L, \lim }$ (Figure 5) from the standard value to the high-performance value due to the reduced thickness of the TPS improves the maximum achievable convenience (by approximately $2 \%$ ). 
Table 7. HEO-LEO transfer, scenarios analyzed.

\begin{tabular}{|c|c|c|}
\hline & Combination & Purpose \\
\hline 1 & GEO; PICA; $\varepsilon$ medium; $T_{B L, \text { lim }}$ standard; TSF $=1$ & Reference nominal case \\
\hline 2 & GEO; PICA; $\varepsilon$ medium; $T_{B L, \text { lim }}$ high performance; TSF $=1$ & $T_{B L, \text { lim }}$ influence \\
\hline 3 & GEO; PICA; $\varepsilon$ high; $T_{B L, \text { lim }}$ standard; TSF $=1$ & $\varepsilon$ influence \\
\hline 4 & GEO; PICA; $\varepsilon$ medium; $T_{B L, \text { lim }}$ standard; TSF $=2$ & TSF influence \\
\hline 5 & GEO; RDCP; $\varepsilon$ medium; $T_{B L, \text { lim }}$ standard; TSF $=1$ & Material influence \\
\hline 6 & GEO; AVCOAT; $\varepsilon$ medium; $T_{B L, \text { lim }}$ standard; TSF $=1$ & Material influence \\
\hline 7 & HEOH; PICA; $\varepsilon$ medium; $T_{B L, \text { lim }}$ standard; TSF $=1$ & $\mathrm{HEO}$ altitude influence; reference case for $\mathrm{HEOH}$ \\
\hline 8 & HEOH; AVCOAT; $\varepsilon$ medium; $T_{B L, \text { lim }}$ standard; TSF $=1$ & Material influence w.r.t. HEOH \\
\hline 9 & $\mathrm{HEOH}$; PICA; $\varepsilon$ high; $T_{B L, \text { lim }}$ standard; TSF $=1$ & $\varepsilon$ influence w.r.t. $\mathrm{HEOH}$ \\
\hline 10 & $\mathrm{HEOH}$; AVCOAT; $\varepsilon$ high; $T_{B L, l i m}$ standard; TSF $=1$ & Material $+\varepsilon$ influence w.r.t. $\mathrm{HEOH}$ \\
\hline 11 & HEOL; PICA; $\varepsilon$ medium; $T_{B L, l i m}$ standard; TSF $=1$ & HEO altitude influence; reference case for HEOL \\
\hline 12 & HEOL; PICA; $\varepsilon$ high; $T_{B L, l i m}$ standard; TSF $=1$ & $\varepsilon$ influence w.r.t. HEOL \\
\hline
\end{tabular}

Legend: in red: GEO nominal case and changes w.r.t. it; in blue: HEOH nominal case and changes w.r.t. it; in green: HEOL nominal case and changes w.r.t. it.

Table 8. Propulsive impulses in the more convenient maneuvers for " $a$ " cases.

\begin{tabular}{cccc}
\hline Case & $\Delta V_{1}(\mathrm{~m} / \mathrm{s})$ & $\Delta V_{2}(\mathrm{~m} / \mathrm{s})$ & $\Delta V_{3}(\mathrm{~m} / \mathrm{s})$ \\
\hline $1 \mathrm{a}$ & 1530.00 & 1.38 & 334.75 \\
$2 \mathrm{a}$ & 1530.00 & -1.83 & 332.91 \\
$3 \mathrm{a}$ & 1500.00 & -2.21 & 375.26 \\
$4 \mathrm{a}$ & 1520.00 & 0.69 & 526.53 \\
$5 \mathrm{a}$ & - & - & - \\
$6 \mathrm{a}$ & 1520.00 & -0.95 & 502.54 \\
$7 \mathrm{a}$ & 1480.00 & 2.86 & 366.44 \\
$8 \mathrm{a}$ & 1470.00 & -4.00 & 568.53 \\
$9 \mathrm{a}$ & 1450.00 & -3.51 & 423.53 \\
$10 \mathrm{a}$ & 1450.00 & 0.67 & 421.13 \\
$11 \mathrm{a}$ & 1460.00 & -3.56 & 410.11 \\
$12 \mathrm{a}$ & 1440.00 & -2.73 & 267.69 \\
\hline
\end{tabular}

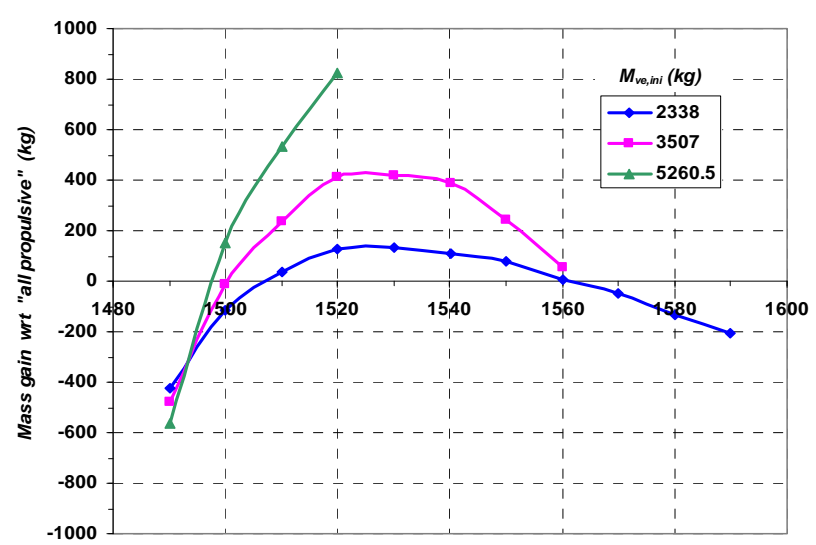

$\Delta V_{1}(\mathrm{~m} / \mathrm{s})$

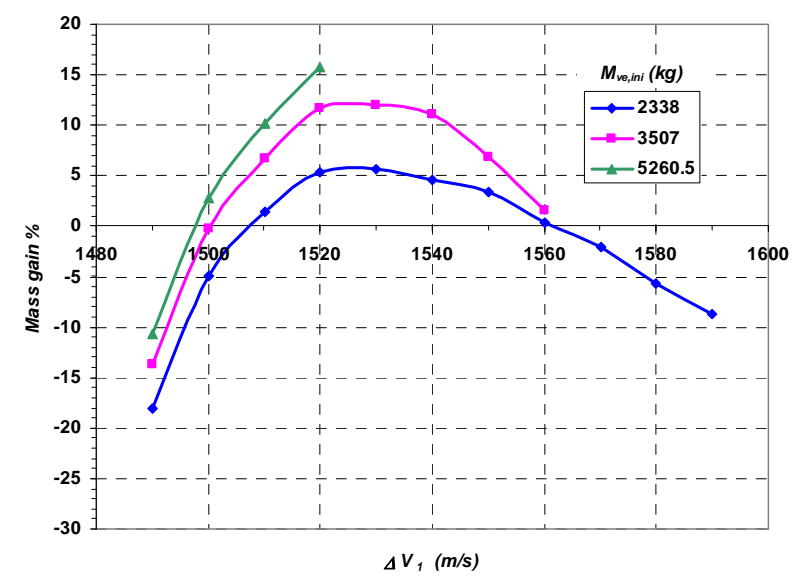

Figure 4. HEO-LEO scenario 1: reference nominal case. 
- The increase in the aerodynamic efficiency of the vehicle (Figure 6) involves a significant extension of the range of feasibility, but a more moderate increase in the range of convenience. Cases with higher $M / S$ benefit most. Even here, the maximum achievable convenience increases (approximately 2\% more).

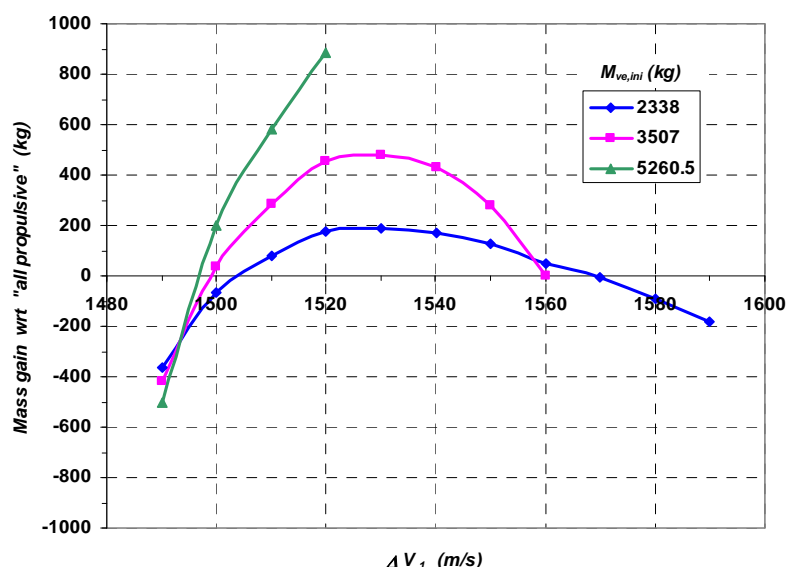

- The doubling of the thermal safety factor (Figure 7), which increases the mass of the TPS, represents a significant performance penalty for the aeroassisted maneuver, which remains affordable for a short interval of $\Delta V_{1}$ only for the case with highest $M / S$.

- The use of other ablative materials (Figures 8 and 9),

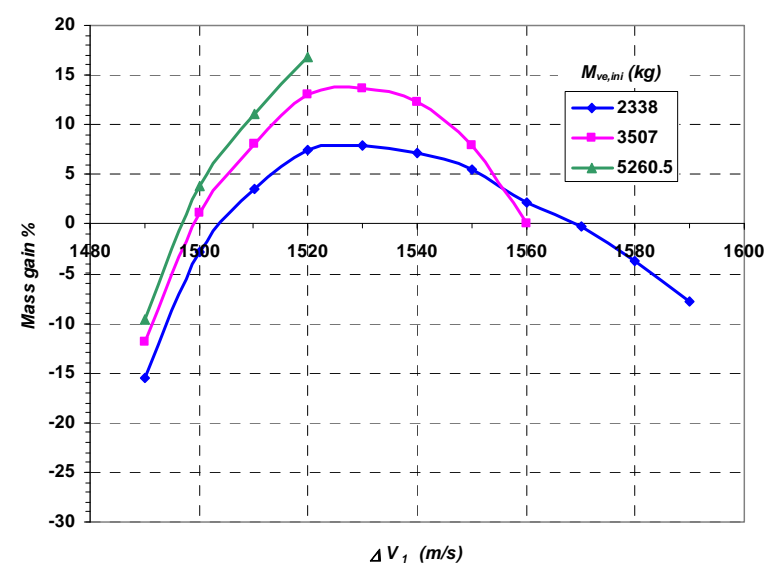

Figure 5. HEO-LEO scenario 2: $T_{B L, \text { lim }}$ influence.
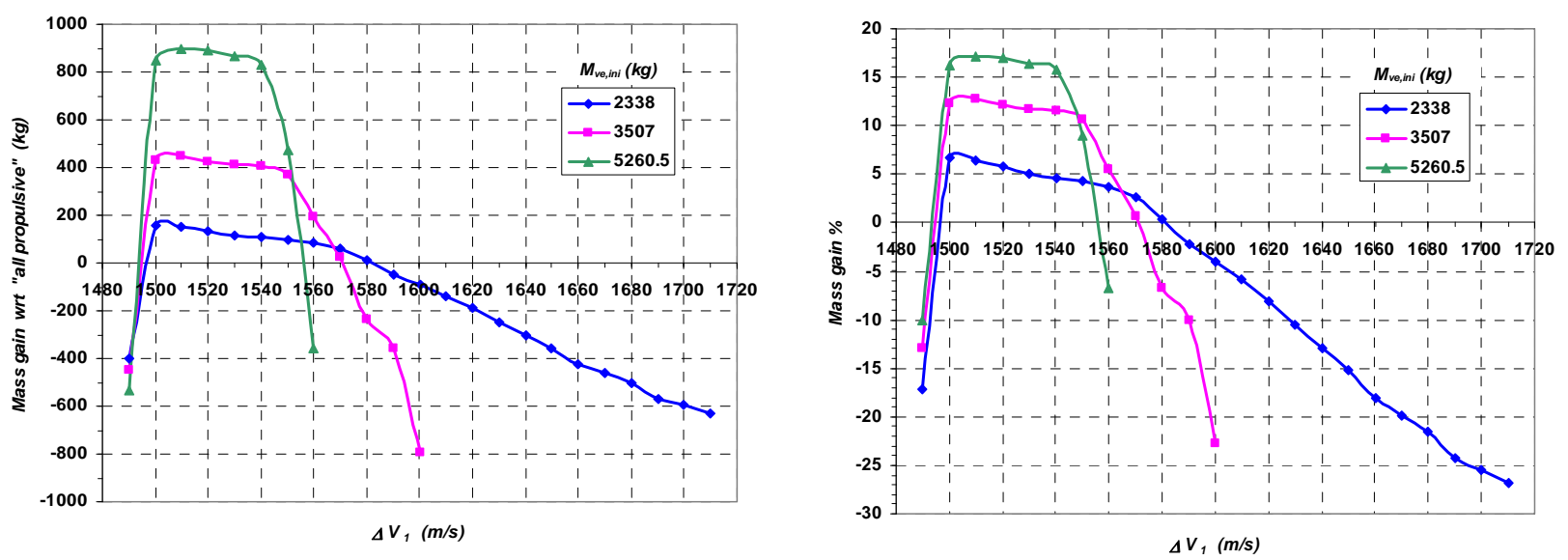

Figure 6. HEO-LEO scenario 3: $\varepsilon$ influence.
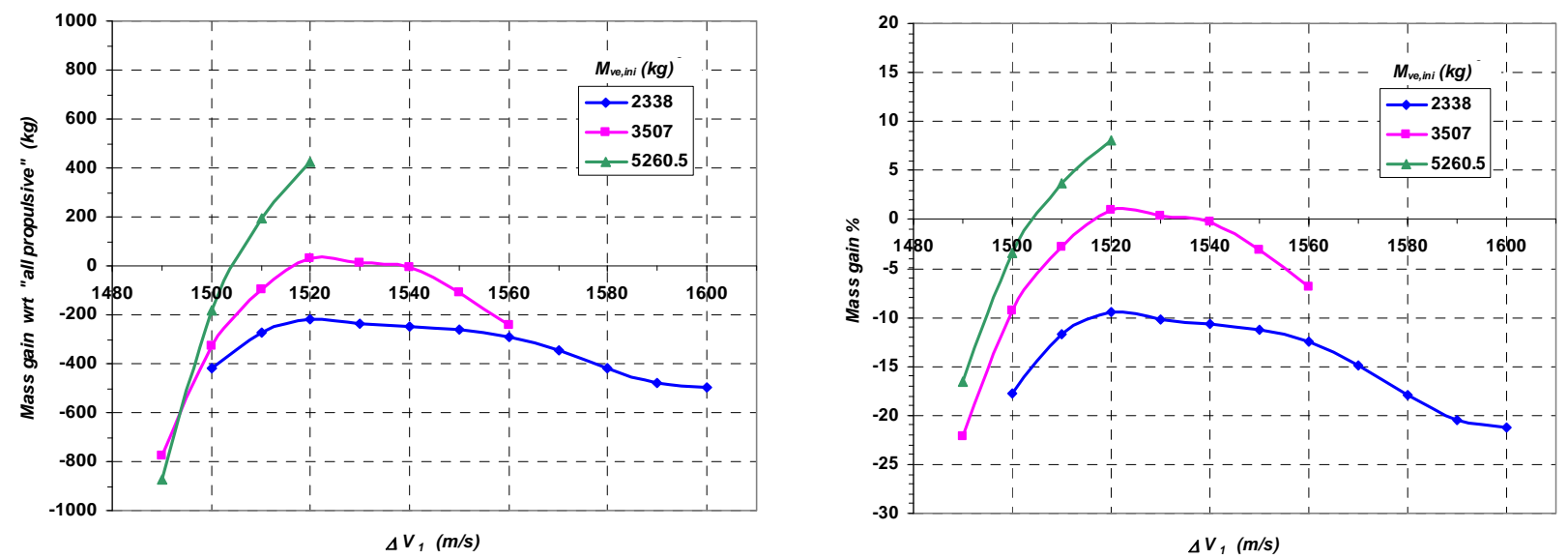

Figure 7. HEO-LEO scenario 4: TSF influence. 

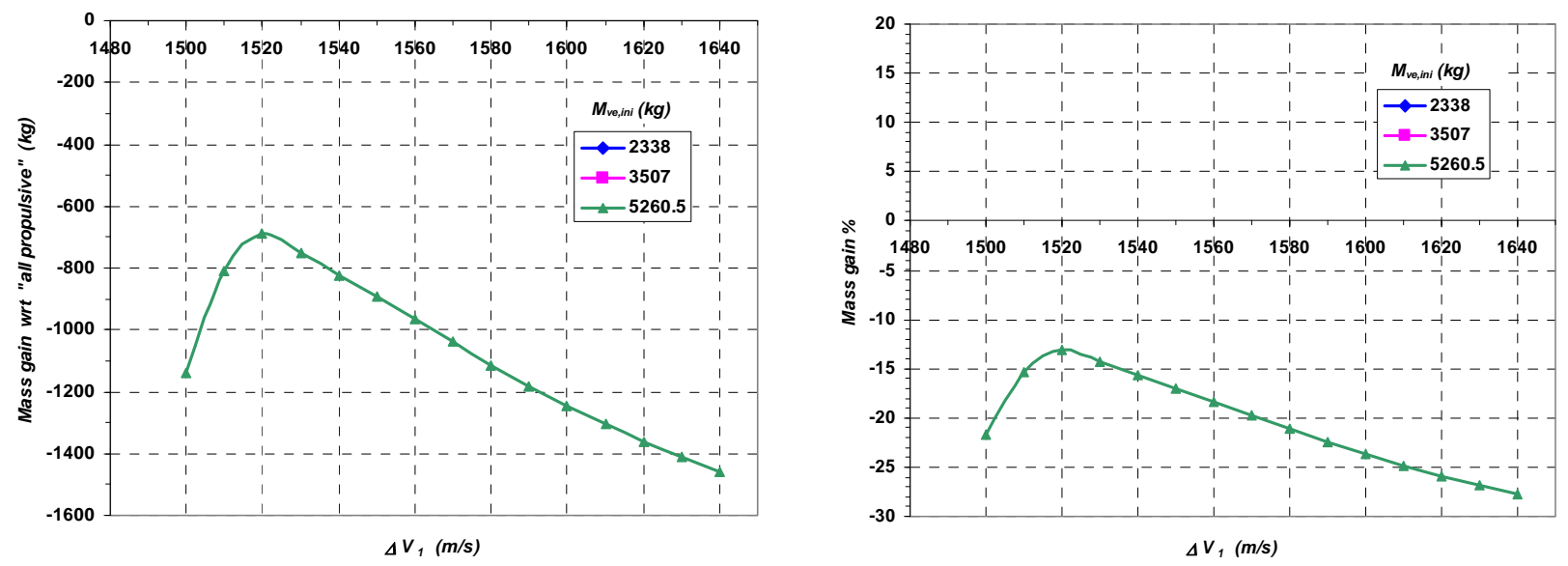

Figure 8. HEO-LEO scenario 5: material (RDCP) influence.
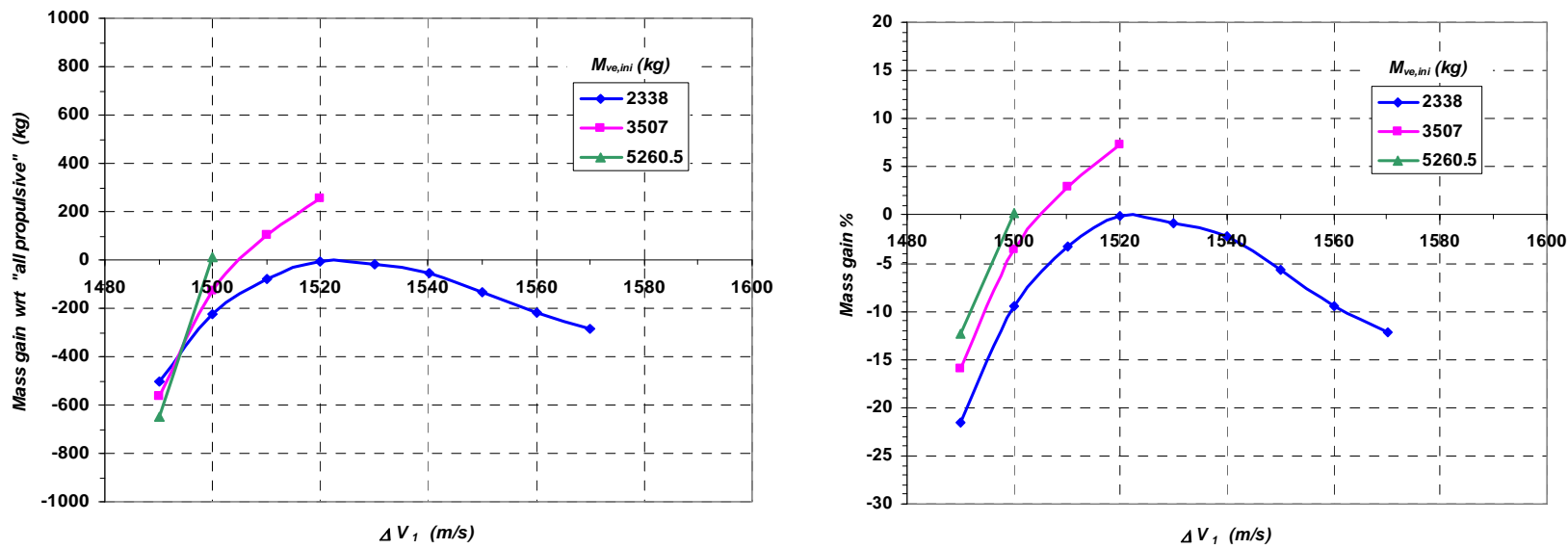

Figure 9. HEO-LEO scenario 6: material (AVCOAT) influence.

compared with the reference material PICA, appears to be inappropriate for a GEO initial altitude. Using the RDCP, the sole case with the highest $M / S$ is feasible, but still not convenient. Conversely, using the AVCOAT increases the feasibility compared with RDCP but limits convenience compared with PICA. Thus, the relatively low density of the PICA is a discriminating element in the selection of the ablative material. Actually, these results are conservative because of the assumption that the vehicle is covered with a TPS with a uniform initial thickness.

In Figure 8, all runs with load values of $200 \mathrm{~kg} / \mathrm{m}^{2}$ and $300 \mathrm{~kg} / \mathrm{m}^{2}$ give results, but the curves are not represented because the final "useful" masses (i.e., all the masses that are not propellant and TPS) are negative. Thus, the vehicle is able to get out of the atmosphere, but without enough fuel to complete the mission.

- Figures 10 (HEO high altitude) and 14 (HEO low altitude) show that the aeroassisted maneuver is much more convenient if the HEO altitude is higher. Lowering the initial altitude reduces the convenience but considerably extends the range of feasibility. The reduced convenience at low altitudes is due to the fact that the Hohmann transfer requires a lower fuel quantity with the lowered the initial altitude for the same final LEO. In contrast, for the corresponding aeroassisted maneuver, even if the TPS is less consistent, the propulsive contribution remains significant.

- Figures 10-13, considered in pairs (the first two and the second two), provide a performance comparison between PICA and AVCOAT, respectively, for high initial orbits in the case of low and high aerodynamic efficiency. The comparison is in favor of the use of PICA, albeit less marked than for GEO orbits. For high orbits for a vehicle with high $\varepsilon$, the performance of PICA remains considerable; however, the performance of AVCOAT, although lower than PICA, is notable. In this context, it seems appropriate to comment on NASA's recent evaluation concerning the choice of material for ablative TPS of the Orion spacecraft. The final decision was between PICA and AVCOAT, and NASA ultimately chose the latter. PICA has been used to date only on a re-entry capsule of the Stardust probe, while AVCOAT has a long history of reliability (Apollo missions). Though the 
results of the present work provide evidence of the tangible prevalence of the PICA performance, the reliability of AVCOAT most likely outweighs these performance considerations.

- The increase in aerodynamic efficiency has less impact in the case of lower orbits (Figures 14 and 15).
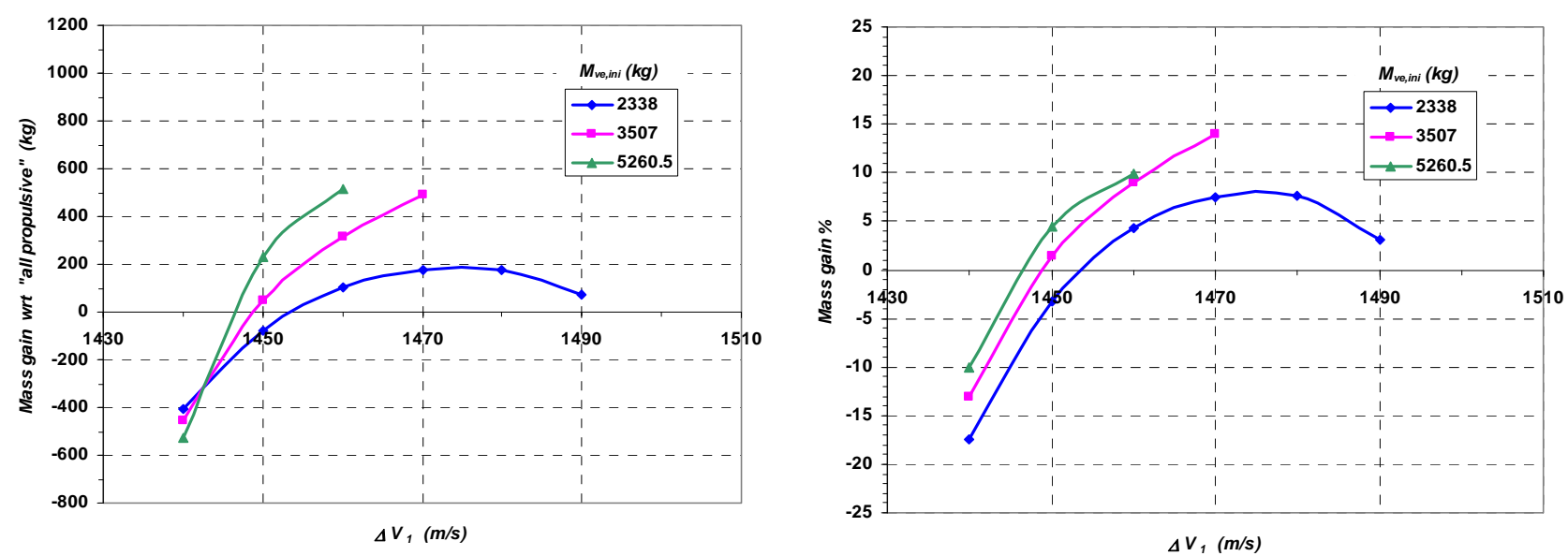

Figure 10. HEO-LEO scenario 7: HEO altitude influence; reference case for HEOH.
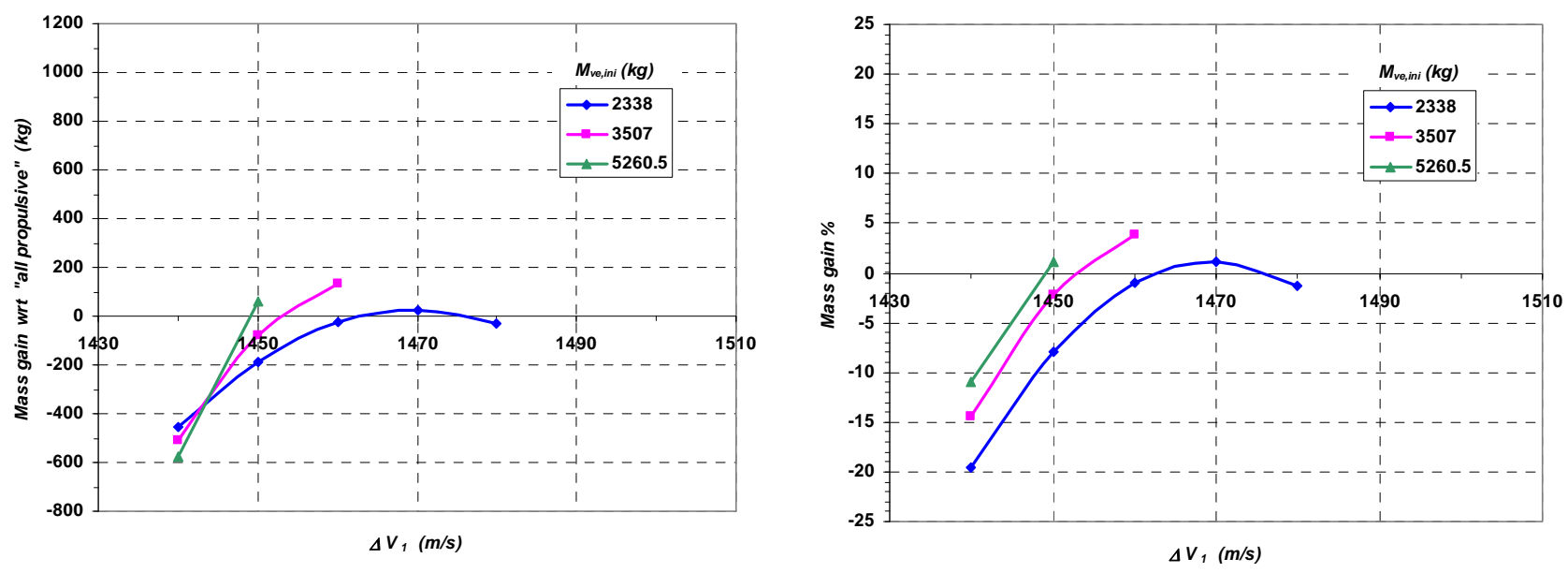

Figure 11. HEO-LEO scenario 8: material (AVCOAT) influence w.r.t. HEOH.
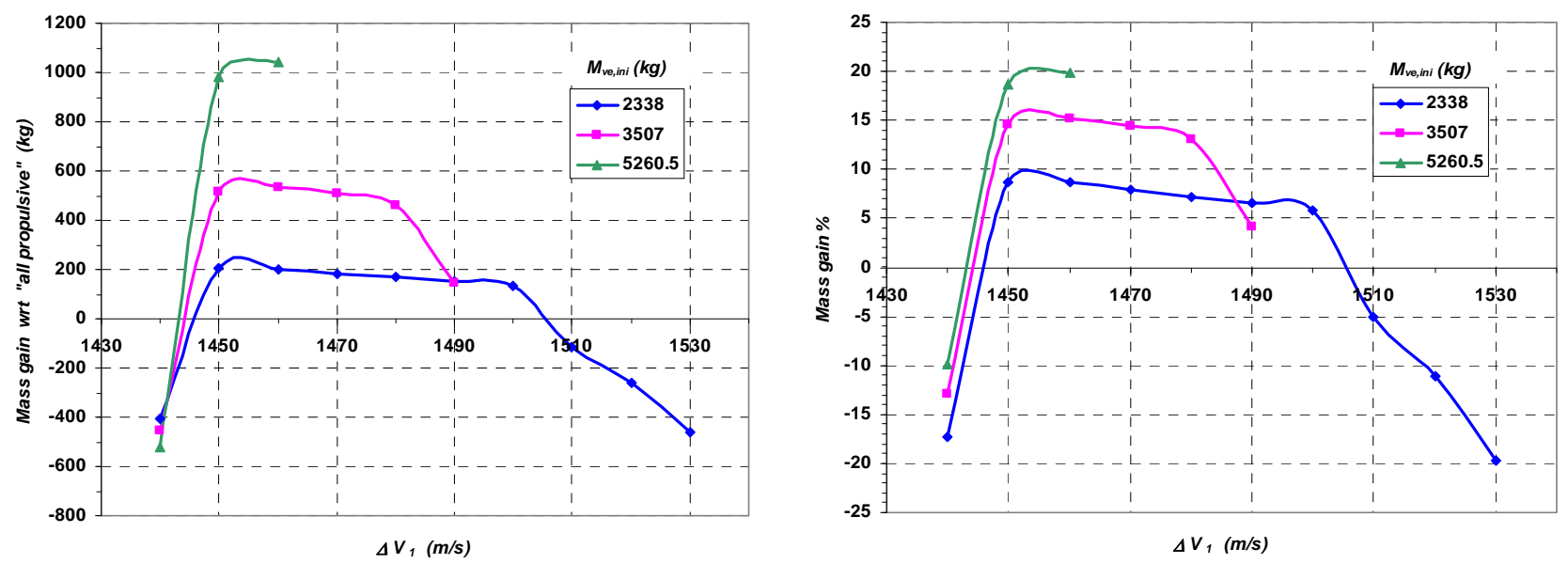

Figure 12. HEO-LEO scenario 9: $\varepsilon$ influence w.r.t. HEOH. 


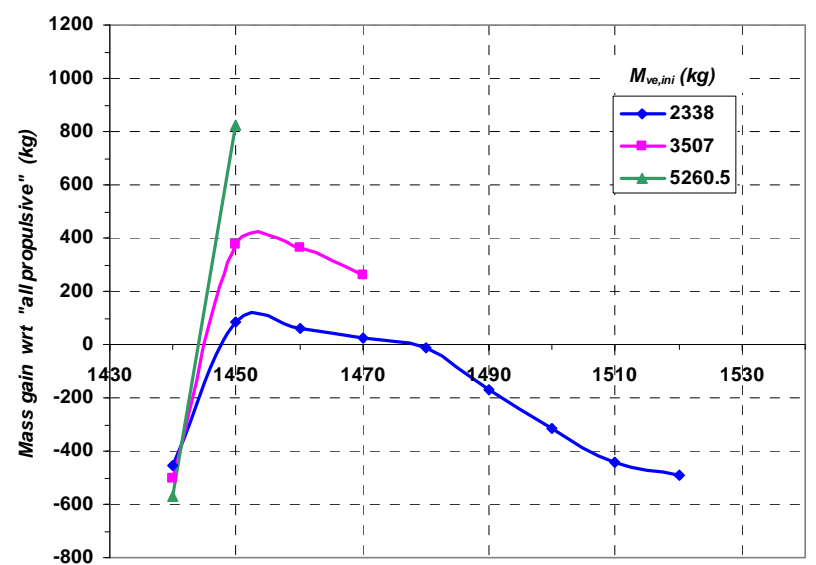

$\Delta v_{1}(\mathrm{~m} / \mathrm{s})$

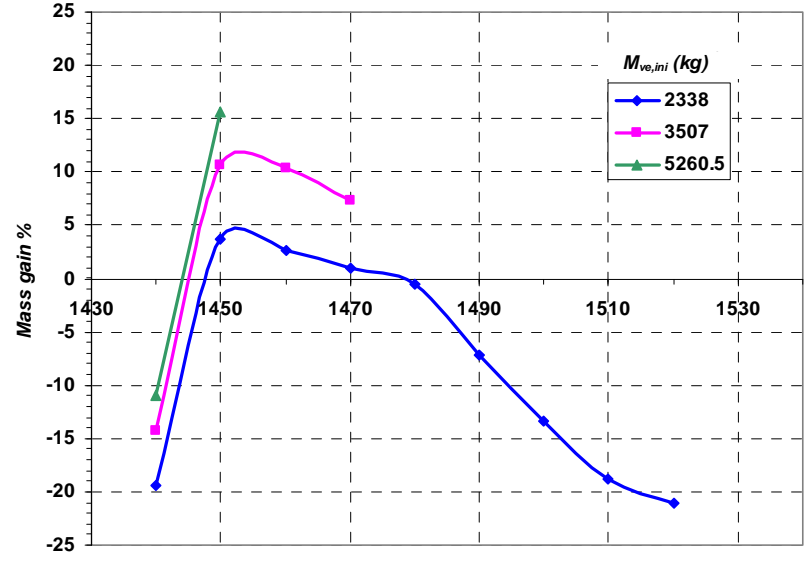

$\Delta v_{1}(m / s)$

Figure 13. HEO-LEO scenario 10: Material (AVCOAT) $+\varepsilon$ influence w.r.t. HEOH.
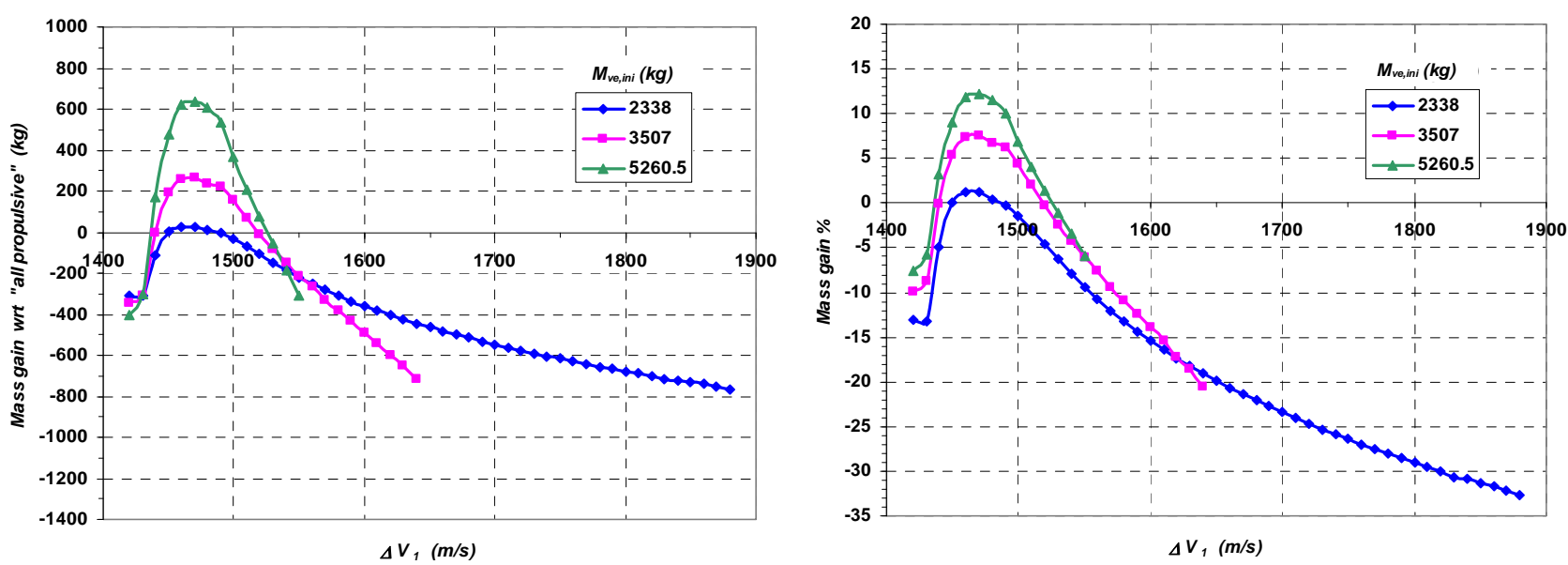

Figure 14. HEO-LEO scenario 11: HEO altitude influence; reference case for HEOL.
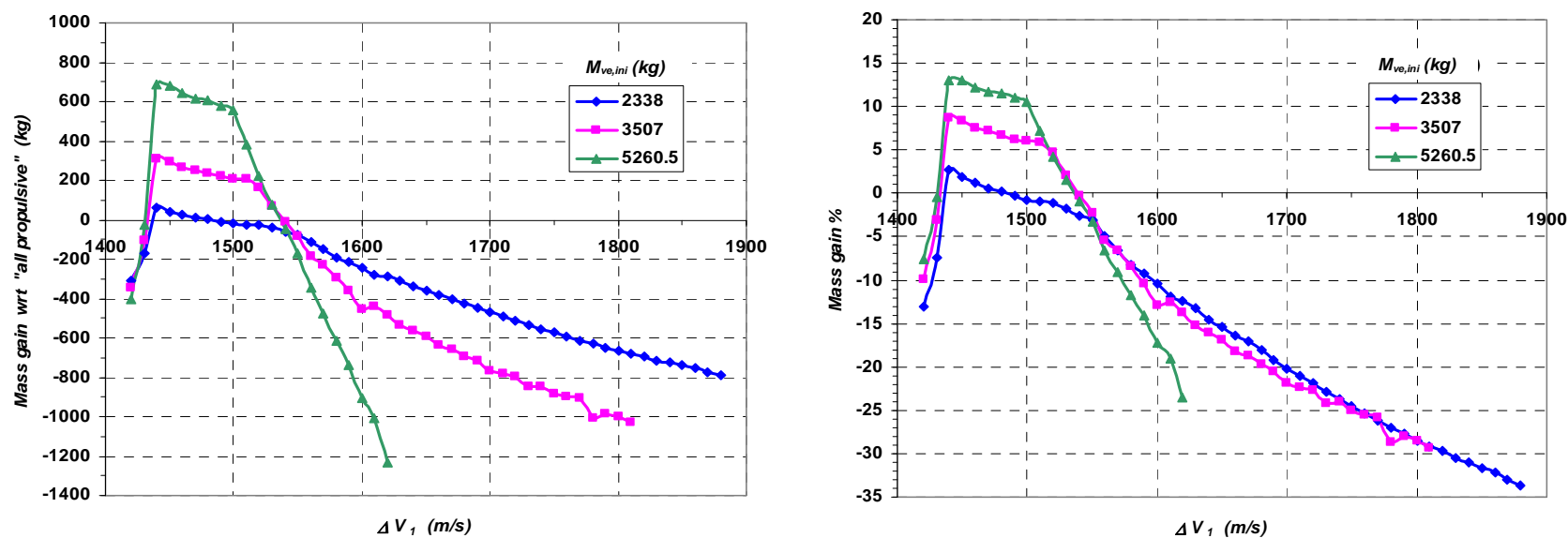

Figure 15. HEO-LEO scenario 12: $\varepsilon$ influence w.r.t. HEOL.

\section{Summary and Conclusions}

Some aeroassisted transfers between coplanar orbits were analyzed to examine the influence of the aerodynamic characteristics of a spacecraft and the characteristics of its heat shield on the maneuver's final performance.

The study was conducted with an original tool developed by the authors comprising highly representative models for thermal analysis, atmospheric flight dynamics, and an optimizer based on a genetic algorithm. The con- 
venience of the maneuver was evaluated by comparison with the corresponding extra-atmospheric Hohmann transfer with two impulses.

The results of the case study indicated that there is a range of feasibility for the maneuver, depending on the first propulsive impulse of deorbit, within which there is, in turn, an interval in which the maneuver is more convenient than the homologous Hohmann transfer in terms of the total mass savings achieved (TPS and propellant). In particular, the results show that in terms of the convenience of the aeroassisted maneuver, the influence of the ablative material type used (low-density advantage) and of the aerodynamic efficiency is the most relevant, whereas other characteristics play a minor role. Significant benefits can be obtained from a careful thermal design, with a suitable thermal safety factor and high-performance adhesives for the bond-line.

For all cases, the highest convenience, and consequently the corresponding optimal trajectories, is obtained when it is not necessary to apply the second propulsion impulse. The most important future development for this type of analysis is increasing the number of cases analyzed in terms of materials, types of maneuvers and spacecraft characteristics.

\section{REFERENCES}

[1] A. Mazzaracchio and M. Marchetti, "A Probabilistic Sizing Tool and Monte Carlo Analysis for Entry Vehicle Ablative Thermal Protection Systems," Acta Astronautica, Vol. 66, No. 5-6, 2010, pp. 821-835. doi:10.1016/j.actaastro.2009.08.033

[2] A. Mazzaracchio and M. Marchetti, "Coupled Aeroassisted Orbital Plane Change Manoeuvre and Thermal Protection System Optimisation," $61^{\text {st }}$ International Astronautical Congress, Prague, September 27-October 1, 2010.

[3] P. Charbonneau and B. Knap, “A User'S Guide to Pikaia 1.0," Boulder, Colorado, 1995.

[4] P. Charbonneau, "An Introduction to Genetic Algorithms for Numerical Optimization," Boulder, Colorado, 2002.

[5] P. Charbonneau, "Release Notes for Pikaia 1.2," Boulder, Colorado, 2002.

[6] C. Gogu, T. Matsumura, R. T. Haftka and A. V. Rao, "Aeroassisted Orbital Transfer Trajectory Optimization Considering Thermal Protection System Mass," Journal of Guidance, Control and Dynamics, Vol. 32, No. 3, 2009, pp. 927-938. doi:10.2514/1.37684

[7] NASA Marshall Space Flight Center, "X-37 Demonstrator to Test Future Launch Technologies in Orbit and Reentry Environments," NASA Facts, May 2003, FS-2003-0565-MSFC

[8] Y. Y. Shi and D. H. Young, "Minimum Fuel Coplanar Aeroassisted Orbital Transfer Using Collocation and Nonlinear Programming," Flight Mechanics/Estimation Theory Symposium, NASA Goddard Space Flight Center, 1991, pp. 461-480 (SEEN92-1407005-13).

[9] US Standard Atmosphere, US Government Printing Office, Washington DC, 1976.

[10] T. J. Collins, W. M. Congdon, S. S. Smeltzer and K. S. Whitley, "High-Temperature Structures, Adhesives, and Advanced Thermal Protection Materials for Next-Generation Aeroshell Design," NASA Langley Research Center, 2006, Paper 2M-02-2005. 


\section{Nomenclature}

$C_{D 0} \quad$ zero-lift drag coefficient

$C_{L, \alpha} \quad$ derivative of $C_{L}$ w.r.t. the angle of attack

$C_{L \text { max }}$ maximum lift coefficient

ff fitness function

$g_{0} \quad$ gravitational acceleration at sea level $\left(\mathrm{m} / \mathrm{s}^{2}\right)$

$H_{A} \quad$ altitude of the initial LEO (m)

$H_{a t m} \quad$ altitude of the sensible atmosphere (m)

$H_{B} \quad$ altitude of the final LEO (m)

$I_{s p} \quad$ propellant specific impulse (s)

$K_{D} \quad$ induced drag factor

$l_{v e} \quad$ vehicle length $(\mathrm{m})$

$m_{T P S, \text { los }}$ TPS mass lost during atmospheric pass $(\mathrm{kg})$

$m_{v e, \text { fin }}$ final vehicle mass $(\mathrm{kg})$

$m_{v e, f i n, a p}$ "all propulsive" case final vehicle mass $(\mathrm{kg})$

$m_{v e, i} \quad$ vehicle mass at atmospheric entry $(\mathrm{kg})$

$m_{v e, i n i} \quad$ initial vehicle mass $(\mathrm{kg})$

$m_{v e, u} \quad$ vehicle mass at atmospheric exit $(\mathrm{kg})$

$R_{A} \quad$ initial HEO radius (m)

$R_{\text {atm }} \quad$ radius of the sensible atmosphere (m)

$R_{B} \quad$ final LEO radius (m)

$R_{f, j^{\prime}} \quad$ reward factor for component " $j$ "

$R_{\oplus} \quad$ Earth's radius (m)

$r_{b} \quad$ vehicle body radius $(\mathrm{m})$

$S \quad$ vehicle reference surface $\left(\mathrm{m}^{2}\right)$

$S_{T P S, v e}$ vehicle TPS total surface $\left(\mathrm{m}^{2}\right)$

$T_{B L} \quad$ bond-line temperature (K)

$T_{B L \text {, lim }}$ bond-line limit temperature (K)

$T S F$ thermal safety factor

$V \quad$ velocity modulus $(\mathrm{m} / \mathrm{s})$

$V_{A} \quad$ circular orbit speed in initial HEO $(\mathrm{m} / \mathrm{s})$

$V_{B} \quad$ circular orbit speed in final LEO $(\mathrm{m} / \mathrm{s})$
$V_{i} \quad$ speed at atmospheric entry $(\mathrm{m} / \mathrm{s})$

$V_{u} \quad$ speed at atmospheric exit $(\mathrm{m} / \mathrm{s})$

$w_{j^{\prime}} \quad$ multiplicative weight of component " $j$ "

$w c_{v e} \quad$ vehicle wing cord (m)

$w s_{v e} \quad$ vehicle wing span (m)

$\Delta V \quad$ speed variation, impulse $(\mathrm{m} / \mathrm{s})$

$\Delta V_{A} \quad$ first impulse for Hohmann transfer $(\mathrm{m} / \mathrm{s})$

$\Delta V_{B} \quad$ second impulse for Hohmann transfer $(\mathrm{m} / \mathrm{s})$

$\Delta V_{A P} \quad$ "all propulsive" (impulse) speed variation $(\mathrm{m} / \mathrm{s})$

$\Delta V_{1} \quad$ deorbit (impulse) speed variation $(\mathrm{m} / \mathrm{s})$

$\Delta V_{L, \text { min }}$ minimum deorbit impulse $(\mathrm{m} / \mathrm{s})$

$\Delta V_{2}$ boost (impulse) speed variation $(\mathrm{m} / \mathrm{s})$

$\Delta V_{3} \quad$ circularizing (impulse) speed variation $(\mathrm{m} / \mathrm{s})$

$\alpha \quad$ angle of attack (rad)

$\varepsilon \quad$ aerodynamic efficiency

$\gamma_{i} \quad$ flight path angle at atmospheric entry (rad)

$\gamma_{u} \quad$ flight path angle at atmospheric exit (rad)

$\mu \quad$ gravitational parameter $\left(\mathrm{m}^{3} / \mathrm{s}^{2}\right)$

$\sigma \quad$ bank angle (rad)

Subscripts

A value at initial HEO

ap "all propulsive"

$B \quad$ value at final LEO

$B L$ bond-line

$H F$ heat flux

$i \quad$ value at atmospheric entry

los lost

$m$ mass

TPS Thermal Protection System

$u \quad$ value at atmospheric exit

ve vehicle 